\title{
Magnetization dynamics in holographic ferromagnets: Landau-Lifshitz equation from Yang-Mills fields
}

\author{
Naoto Yokoi®, ${ }^{1,2}$ Koji Sato, ${ }^{2}$ and Eiji Saitoh ${ }^{1,2,3,4,5}$ \\ ${ }^{1}$ Department of Applied Physics, The University of Tokyo, Tokyo 113-8656, Japan \\ ${ }^{2}$ Institute for Materials Research, Tohoku University, Sendai 980-8577, Japan \\ ${ }^{3}$ Advanced Institute for Materials Research, Tohoku University, Sendai 980-8577, Japan \\ ${ }^{4}$ Center for Spintronics Research Network, Tohoku University, Sendai 980-8577, Japan \\ ${ }^{5}$ Advanced Science Research Center, Japan Atomic Energy Agency, Tokai 319-1195, Japan
}

(Received 23 August 2019; published 22 November 2019)

\begin{abstract}
We introduce a new approach to understand magnetization dynamics in ferromagnets based on the holographic realization of ferromagnets. A Landau-Lifshitz equation describing the magnetization dynamics is derived from a Yang-Mills equation in the dual gravitational theory, and temperature dependences of the spin-wave stiffness and spin transfer torque appearing in the holographic Landau-Lifshitz equation are investigated by the holographic approach. The results are consistent with the known properties of magnetization dynamics in ferromagnets with conduction electrons.
\end{abstract}

DOI: 10.1103/PhysRevD.100.106012

\section{INTRODUCTION}

The Landau-Lifshitz equation [1] is the fundamental equation for describing the dynamics of magnetization (density of magnetic moments) in various magnetic materials. It has been also playing a fundamental role in the development of modern spintronics [2]: For instance, its extension to the coupled systems of localized magnetic moments and conduction electrons has led to the concepts of spin transfer torque [3,4] and spin pumping [5]. So far, the symmetries and reciprocity in electronic systems have been the guiding principles to develop such extensions. In this article, we introduce another guiding principle to explore the new extensions and magnetization dynamics on the basis of the holographic duality.

The holographic duality is the duality between the quantum many body system defined in $d$-dimensional space-time and the gravitational theory (with some matter fields) which lives in $(d+1)$-dimensional space-time [6-8]. ${ }^{1}$ We constructed a holographic dual model of three-dimensional ferromagnetic systems, which exhibits the ferromagnetic phase transition with spontaneous magnetization and the consistent magnetic properties at low

\footnotetext{
${ }^{1}$ See Refs. $[9,10]$ for recent reviews on the applications of the holographic duality to condensed matter physics.

Published by the American Physical Society under the terms of the Creative Commons Attribution 4.0 International license. Further distribution of this work must maintain attribution to the author(s) and the published article's title, journal citation, and DOI. Funded by SCOAP.
}

temperatures [11]..$^{2}$ In the holographic duality, finite temperature effect in ferromagnetic systems can be incorporated as the geometrical effect of black holes in higher dimensional bulk gravity, and the Wick rotation at finite temperatures is not required for the analysis in the dual gravitational theory. Thus, the novel analysis for real-time dynamics of quantum many body systems in nonequilibrium situations can be performed using the holographic approach (for a review, see Ref. [15]). In addition, the holographic duality is known to be a strong-weak duality, which relates strongly correlated quantum systems to classical gravitational theories. From these viewpoints, the holographic approach can provide new useful tools to analyze nonequilibrium and nonlinear dynamics of magnetization in ferromagnets.

In ferromagnets, spin currents are generated by magnetization dynamics. From the holographic dictionary between the quantities of ferromagnets and gravitational theory [11], the spin currents in ferromagnets correspond to the $S U(2)$ gauge fields in the dual gravitational theory. This correspondence indicates that the dynamics of spin currents, consequently the dynamics of magnetization, can be described by the Yang-Mills equation for $S U(2)$ gauge fields [16] in the holographic dual theory. In the following, we derive a Landau-Lifshitz equation for magnetization dynamics from the Yang-Mills equation within the holographic realization of ferromagnets. This derivation can provide novel perspectives for magnetization dynamics from the non-Abelian gauge theory.

\footnotetext{
${ }^{2}$ Other holographic approaches to ferromagnetic systems have been also discussed in Refs. [12-14].
} 
TABLE I. Holographic dictionary between the dual gravitational theory and ferromagnets.

\begin{tabular}{ccc}
\hline \hline Dual gravity & & Ferromagnet \\
\hline Scalar field $\phi^{a}$ & $\Leftrightarrow$ & Magnetization $M^{a}$ \\
$S U(2)$ gauge field $A_{M}^{a}$ & $\Leftrightarrow$ & Spin current $J_{s \mu}^{a}$ \\
$U(1)$ gauge field $B_{M}$ & $\Leftrightarrow$ & Charge current $J_{\mu}$ \\
Metric $g_{M N}$ & $\Leftrightarrow$ & Stress tensor $T_{\mu \nu}$ \\
\hline \hline
\end{tabular}

This article is organized as follows. In Sec. II, we summarize the results of the magnetic properties obtained from the holographic realization of ferromagnets in thermodynamic equilibrium. An extension to nonequilibrium situation including the fluctuations of magnetization and spin currents is discussed in the dual gravitational theory, and the holographic equation of magnetization dynamics is derived in Sec. III. In Sec. IV, temperature dependences of the parameters in the resulting holographic equation are investigated by numerical calculations. Finally, we summarize the results in Sec. V.

\section{HOLOGRAPHIC DUAL MODEL OF FERROMAGNETS}

We begin with a brief summary on the holographic dual model of three-dimensional ferromagnets [11]. The dual model is the five-dimensional gravitational theory with an $S U$ (2) gauge field $A_{M}^{a}$ and a $U(1)$ gauge field $B_{M}$, whose action is given by

$$
\begin{aligned}
S= & \int \sqrt{-g} d^{5} x\left[\frac{1}{2 \kappa^{2}}(R-2 \Lambda)-\frac{1}{4 e^{2}} G_{M N} G^{M N}\right. \\
& \left.-\frac{1}{4 g^{2}} F_{M N}^{a} F^{a M N}-\frac{1}{2}\left(D_{M} \phi^{a}\right)^{2}-V(|\phi|)\right] .
\end{aligned}
$$

Here, $R$ is the scalar curvature of space-time, and the field strength is defined by $F_{M N}^{a}=\partial_{M} A_{N}^{a}-\partial_{N} A_{M}^{a}+\epsilon^{a b c} A_{M}^{b} A_{N}^{c}$ and $G_{M N}=\partial_{M} B_{N}-\partial_{N} B_{M}$, respectively. The index $a$ labels spin directions in the $S U(2)$ space $(a=1 \sim 3)$, the index $M$ labels space-time directions in five dimensions $(M, N=0 \sim 4)$, and $\epsilon^{a b c}$ is a totally antisymmetric tensor with $\epsilon^{123}=1$. The model also includes a triplet scalar field $\phi^{a}$ with the covariant derivative $D_{M} \phi^{a}=\partial_{M} \phi^{a}+$ $\epsilon^{a b c} A_{M}^{b} \phi^{c}$, and the $S U(2)$-invariant scalar potential $V(|\phi|)$ with the norm $|\phi|^{2}=\sum_{a=1}^{3}\left(\phi^{a}\right)^{2}$. Note that the scalar field is neutral under the $U(1)$ gauge transformation. In order to guarantee asymptotic anti-de Sitter (AdS) backgrounds, the negative cosmological constant $\Lambda=-6 / \ell^{2}$ is introduced. The field-operator correspondence in the holographic duality $[7,8]$ leads to the holographic dictionary between the fields of the dual gravitational theory and the physical quantities of ferromagnets in Table I.

\section{A. Black hole as heat bath}

In order to establish the holographic dictionary, thermodynamical properties of the physical quantities of ferromagnets should be calculated in the dual gravitational theory. In Ref. [11], the temperature dependences of magnetic quantities and the behavior of ferromagnetic phase transition are thoroughly discussed. In the context of the holographic duality, finite temperature effects in the ferromagnets can be incorporated by introducing the black holes into the dual gravitational theory as the background space-time. Indeed, the dual gravitational theory has the charged black hole solution which is a solution to the Einstein, Yang-Mills, and Maxwell equations derived from the action (1):

$$
\begin{aligned}
R_{M N} & +\left(\Lambda-\frac{1}{2} R\right) g_{M N} \\
= & \frac{\kappa^{2}}{2 e^{2}}\left(2 G_{K M} G^{K}{ }_{N}-\frac{1}{2} G_{K L} G^{K L} g_{M N}\right) \\
& +\frac{\kappa^{2}}{2 g^{2}}\left(2 F_{K M}^{a} F^{a K}{ }_{N}-\frac{1}{2} F_{a K L} F^{a K L} g_{M N}\right),
\end{aligned}
$$

$\nabla_{M} F^{a M N}+\epsilon^{a b c} A_{M}^{b} F^{c M N}=0, \quad$ and $\quad \nabla_{M} G^{M N}=0$,

where $\nabla_{M}$ is the covariant derivative for the affine connection, and the space-time indices $M, N$ are raised or lowered by the bulk metric $g_{M N}$. Here, we neglect the contribution from the scalar field and set $\phi^{a}=0$ for the background. The metric of the black hole ${ }^{3}$ is given by

$$
\begin{aligned}
d s^{2} & =g_{M N} d x^{M} d x^{N} \\
& =\frac{r^{2}}{\ell^{2}}\left(-f(r) d t^{2}+d x^{2}+d y^{2}+d z^{2}\right)+\frac{\ell^{2}}{f(r)} \frac{d r^{2}}{r^{2}},
\end{aligned}
$$

with the radial function,

$$
f(r)=1-\left(1+Q^{2}\right)\left(\frac{r_{H}}{r}\right)^{4}+Q^{2}\left(\frac{r_{H}}{r}\right)^{6} .
$$

Here, we define the parameter $Q$ :

$$
Q^{2}=\frac{2 \kappa^{2}}{3}\left(\frac{\mu_{e}^{2}}{e^{2}}+\frac{\mu_{s}^{2}}{g^{2}}\right)
$$

The $U(1)$ charge $\mu_{e}$ and $S U(2)$ charge $\mu_{s}$ of the black hole are supported by the time components of the gauge fields:

\footnotetext{
${ }^{3}$ This type of non-Abelian black holes has been discussed in the context of the holographic duality, in the literature such as Refs. [12,17].
} 


$$
\begin{aligned}
& B_{0}=\mu_{e}\left(\frac{r_{H}}{\ell}\right)\left(1-\frac{r_{H}^{2}}{r^{2}}\right), \\
& A_{0}^{3}=\mu_{s}\left(\frac{r_{H}}{\ell}\right)\left(1-\frac{r_{H}^{2}}{r^{2}}\right) .
\end{aligned}
$$

Note that the black hole solution (4) is asymptotically AdS at $r \rightarrow \infty$, and has the (outer) horizon $r=r_{H}$.

For the following discussion, we make a coordinate change of the radial coordinate $r$ into $u$ by $u=1 / r$, and the black hole metric becomes

$d s^{2}=\frac{1}{u^{2}}\left(-f(u) d t^{2}+d x^{2}+d y^{2}+d z^{2}+\frac{d u^{2}}{f(u)}\right)$,

and the transformed function $f(u)$ is given by

$$
f(u)=1-\left(1+Q^{2}\right) u^{4}+Q^{2} u^{6},
$$

where we have set the gravitational parameters, $\kappa=r_{H}=$ $\ell=1$, for simplicity.

In the holographic dual model, the black hole (8) plays the role of the heat bath; due to the Hawking radiation, the black hole temperature is given by

$$
T=\frac{2-Q^{2}}{2 \pi}
$$

and the calculations on the black hole background lead to the thermodynamical properties of the corresponding ferromagnet. Since we focus only on the dynamics of magnetization and spin current, the background space-time is fixed to be the black hole metric (8) in the following.

\section{B. Thermodynamics of ferromagnets from scalar dynamics on charged black hole}

In order to investigate the thermodynamics of magnetization, we examine the equation of motion for the scalar field $\phi^{a}$, which is also derived from the action (1):

$$
\frac{1}{\sqrt{-g}} \partial_{M}\left(\sqrt{-g} D^{M} \phi^{a}\right)+\varepsilon^{a b c} A_{M}^{b} D^{M} \phi^{c}=\frac{\partial V}{\partial \phi^{a}} .
$$

Here, we consider a static and homogeneous solution in the boundary coordinates, $x^{\mu}=\left(t, x^{1}, x^{2}, x^{3}\right)$, which corresponds to the homogeneous magnetization in ferromagnets. Without loss of generality, the ansatz for such a scalar field, which is invariant under the translations on the boundary, is given by

$$
\phi^{1}=\phi^{2}=0, \quad \phi^{3}=\Phi(u) \neq 0 .
$$

Inserting this ansatz, the metric (8), and the gauge fields (7) into the Eq. (11), we obtain the following equation for $\Phi(u)$ :

$$
u^{2} f(u) \frac{d^{2} \Phi}{d u^{2}}+\left(u^{2} \frac{d f(u)}{d u}-3 u f(u)\right) \frac{d \Phi}{d u}=\frac{\partial V}{\partial \Phi} .
$$

This equation governs the thermodynamics of magnetization in the dual gravitational theory. We can analyze the solution to this equation numerically with a simple quartic potential $V(|\phi|)=\lambda\left(|\phi|^{2}-m^{2} / \lambda\right)^{2} / 4$, and the asymptotic behavior of the numerical solution near the boundary $u \sim 0$ (or $r \sim \infty$ ) is obtained:

$$
\Phi(u) \simeq H_{0} u^{\Delta_{-}}+M(T) u^{\Delta_{+}}
$$

with $\Delta_{ \pm}=2 \pm \sqrt{4-m^{2}}$. According to the standard recipe in the holographic duality [18,19], the coefficients $H_{0}$ and $M(T)$ in the asymptotic expansion correspond to an external magnetic field and a magnetization at temperature $T$ (under $H_{0}$ ), respectively. In Ref. [11], the resulting temperature dependences of magnetization, magnetic susceptibility, and specific heat have been shown to reproduce the ferromagnetic phase transition in the mean field theory. Furthermore, the temperature dependences at low temperatures are also consistent with the existence of the spin wave excitations (magnons) and conduction electrons in low-temperature ferromagnets.

For later convenience, we also comment on the solutions for the gauge fields. Assuming the translational and rotational invariance on the boundary, equilibrium solutions for the gauge fields are given by the following form:

$$
B_{0}=b(u) \quad \text { and } \quad A_{0}^{3}=a^{3}(u),
$$

where all the other components vanish. Inserting this ansatz and (12), the Maxwell and Yang-Mills equations on the black hole are reduced to the following simple forms:

$$
\frac{d}{d u}\left(\frac{1}{u} \frac{d b}{d u}\right)=0 \quad \text { and } \quad \frac{d}{d u}\left(\frac{1}{u} \frac{d a^{3}}{d u}\right)=0 .
$$

The general solutions are given by the forms (7) in terms of $u$,

$$
b(u)=\mu_{e}\left(1-u^{2}\right) \quad \text { and } \quad a^{3}(u)=\mu_{s}\left(1-u^{2}\right) .
$$

Here, we impose the boundary conditions $B_{0}=0$ and $A_{0}^{3}=0$ at the horizon $(u=1)$, which guarantee the regularity of the gauge fields on the horizon. The remaining integral constants, $\mu_{e}$ and $\mu_{s}$, correspond respectively to the electrochemical potential of underlying electrons and the spin chemical potential (or spin voltage), through the holographic dictionary.

To summarize, the solutions (14) and (17) on the charged black hole describe the thermodynamical property of the holographic dual ferromagnets in the equilibrium. 


\section{MAGNETIZATION DYNAMICS IN HOLOGRAPHIC FERROMAGNETS}

In this section, we extend the holographic analysis in the equilibrium, summarized in the previous section, to more general situations including the dynamics of magnetization and spin currents. In order to discuss the dynamics of magnetization and spin currents, the static and homogeneous ansatze for the scalar field (12) and the gauge fields (15) need to be generalized. Here, we focus on the low energy dynamics with the long wave length in the ordered phase (symmetry broken phase) below the Curie temperature, where various phenomena in modern spintronics are intensively studied.

\section{A. Generalized ansatz and effective equations of motion}

For the scalar field, following the standard derivation of the equation for magnetization dynamics, we consider the generalized ansatz for the scalar field as a factorized form:

$\phi^{a}(u, t, x)=\Phi(u) n^{a}(t, x) \quad$ with $\quad \sum_{a=1}^{3} n^{a} n^{a}=1$,

where $\Phi(u)$ is a solution of the equation (13) with the asymptotic behavior (14). Note that, since we focus only on the dynamics of spontaneous magnetization, we fix $H_{0}=0$ throughout this article. In this ansatz, $n^{a}(t, x)$ corresponds to the (local) direction of magnetization in ferromagnets.

In ferromagnetic systems, the magnetization dynamics generates various dynamics of spin currents [2]. In the holographic dual theory, the scalar dynamics is also expected to induce the dynamics of the corresponding $S U$ (2) gauge field, and thus we generalize the static and homogeneous ansatz for the $S U(2)$ gauge fields to the following factorized forms:

$$
\begin{aligned}
A_{0}^{\|}(u, t, x) & =\left(1-u^{2}\right) a_{0}^{\|}(t, x), \\
A_{0}^{\perp}(u, t, x) & =\left(1-u^{2}\right) a_{0}^{\perp}(t, x), \\
A_{i}^{\|}(u, t, x) & =G^{\|}(u) a_{i}^{\|}(t, x), \\
A_{i}^{\perp}(u, t, x) & =G^{\perp}(u) a_{i}^{\perp}(t, x) \quad(i=1 \sim 3),
\end{aligned}
$$

where we set the radial component $A_{u}^{a} \equiv 0$ by using the gauge degrees of freedom. Due to the nontrivial scalar solution $\Phi(u)$, corresponding to the spontaneous magnetization, the $S U(2)$ gauge symmetry is broken to $U(1)$. The gauge fields can be correspondingly decomposed into an unbroken component $A_{\mu}^{\|}$and two broken components $A_{\mu}^{\perp}$, which are defined by $A_{\mu}^{\|} \propto n^{a}$ and $n \cdot A_{\mu}^{\perp}=0$, respectively. As in the case of the static solutions, the time components of gauge fields should satisfy the horizon boundary condition, $A_{0}^{a}=0$ at $u=1$, for the regularity. Although the spatial components $A_{i}^{a}$ are not required to vanish on the horizon, the regularity (or finiteness) at the horizon is required. The asymptotic solutions to the linearized YangMills equation near the boundary $(u \sim 0)$ give the asymptotic expansions for the radial functions $G^{\|}(u)$, and $G^{\perp}(u)$,

$$
\begin{aligned}
G^{\|}(u) & =1-\sigma_{s}^{\|} u^{2}+\mathcal{O}\left(u^{4}\right), \\
G^{\perp}(u) & =1+\sigma_{s}^{\perp} u^{2}+\mathcal{O}\left(u^{4}\right) .
\end{aligned}
$$

We discuss the concrete numerical solutions of $G^{a}(u)$ and their physical implications in the next section.

Since the scalar field $\phi^{a}$ does not have the $U(1)$ charge, the fluctuation (or dynamics) of $\phi^{a}$ does not induce further dynamics for the $U(1)$ gauge field, which implies the solution for $B_{\mu}$ in (7) is unchanged, and we can neglect the dynamics of $B_{\mu}$.

At first, we consider the equation of motion for the scalar field $\phi^{a}$. Inserting the generalized ansatz (18) into the Eq. (11), we obtain the following equation for $n^{a}$ :

$$
\begin{aligned}
& {\left[u^{5} \partial_{u}\left(u^{-3} f(u) \partial_{u} \Phi\right)-\frac{\partial V}{\partial \Phi}\right] n^{a}} \\
& \quad=\left[\frac{u^{2}}{f(u)} D_{t} D_{t} n^{a}-u^{2} D_{i} D_{i} n^{a}\right] \Phi .
\end{aligned}
$$

Here, we have used the gauge condition $A_{u}^{a}=0$, and the gauge covariant derivative is defined as $D_{\mu} n^{a}=\partial_{\mu} n^{a}+$ $\varepsilon^{a b c} A_{\mu}^{b} n^{c}$. The left-hand side of Eq. (21) is proportional to the Eq. (13), and thus vanishes for the solution $\Phi(u)$. Since $\Phi(u)$ is a nontrivial solution, which is not identically zero, we have the effective equation of motion for $n^{a}$ :

$$
f^{-1} D_{t} D_{t} n^{a}-D_{i} D_{i} n^{a}=0 .
$$

Next, the equation of motion for the gauge fields is considered. The Yang-Mills equation for the $S U(2)$ gauge field $A_{M}^{a}$ is derived by the variation of the holographic action (1) and given by

$$
\frac{1}{\sqrt{-g}} \partial_{N}\left(\sqrt{-g} F^{N M a}\right)+\epsilon^{a b c} A_{N}^{b} F^{N M c}=J^{M a},
$$

where the $S U(2)$ current is defined as

$J_{M}^{a}=\varepsilon^{a b c} \phi^{b} D_{M} \phi^{c}=\varepsilon^{a b c} \phi^{b}\left(\partial_{M} \phi^{c}+\varepsilon^{c d e} A_{M}^{d} \phi^{e}\right)$.

Here, we set the gauge coupling parameter, $g=1$, for simplicity. Unlike the static case, the generalized ansatz (18) and (19) give the nonvanishing currents:

$J_{\mu}^{a}=\Phi^{2}\left(\varepsilon^{a b c} n^{b} \partial_{\mu} n^{c}+\varepsilon^{a b c} \varepsilon^{c d e} n^{b} a_{\mu}^{d} n^{e}+\mathcal{O}\left(u^{2}\right)\right)$.

Note that the radial component of the currents still vanishes, $J_{u}^{a} \equiv 0$, due to the gauge fixing condition $A_{u}^{a} \equiv 0$. With this current, we can explicitly write down the Yang-Mills equations on the charged black hole (8), in the boundary direction, 


$$
\begin{gathered}
J_{0}^{a}=u^{3} f \partial_{u}\left(u^{-1} F_{u 0}^{a}\right)+u^{2}\left(D_{i} F_{i 0}^{a}\right), \\
J_{i}^{a}=u^{3} \partial_{u}\left(u^{-1} f F_{u i}^{a}\right)-u^{2} f^{-1}\left(D_{0} F_{0 i}^{a}\right)+u^{2}\left(D_{j} F_{j i}^{a}\right),
\end{gathered}
$$

where the gauge covariant derivative for the field strength is defined as $D_{\mu} F_{\nu \rho}^{a}=\partial_{\mu} F_{\nu \rho}^{a}+\varepsilon^{a b c} A_{\mu}^{b} F_{\nu \rho}^{c}$. Inserting the ansatz (19), the Yang-Mills equations give the equations for $n^{a}$ and $a_{\mu}^{a}$. In summary, using the generalized ansatze, we have obtained the coupled equations of motion for $n^{a}$ and $a_{\mu}^{a},(22),(26)$, and (27).

\section{B. Landau-Lifshitz equation from Yang-Mills equation}

Since it is difficult to find the general solutions for the coupled nonlinear partial differential equations, we seek simple trial solutions for $n^{a}$ and $a_{\mu}^{a}$ to obtain the effective equations of motion. At first, instead of looking for general solutions to the Eq. (22), we consider the solutions to the simpler equations:

$$
D_{t} n^{a}=0 \quad \text { and } \quad D_{i} n^{a}=0
$$

which are explicitly given by

$$
\partial_{\mu} n^{a}+\epsilon^{a b c} a_{\mu}^{b} n^{c}=0+\mathcal{O}\left(u^{2}\right) .
$$

These equations lead to the ground state solutions for the effective Hamiltonian for $n^{a}$ :

$$
\mathcal{H}_{\mathrm{eff}}=\frac{f}{2}\left(\pi^{a}\right)^{2}+\frac{1}{2}\left(D_{i} n^{a}\right)^{2}
$$

where the conjugate momentum is defined by $\pi^{a}=$ $f^{-1} D_{t} n^{a}$. In this article, we wish to discuss the dynamics of magnetization and spin currents in the boundary ferromagnetic system, which is given by the leading terms in the asymptotic expansions at $u \sim 0$. Hence, the higher order terms in the expansion with respect to $u$ are irrelevant, and we neglect them in the following. Dropping the $\mathcal{O}\left(u^{2}\right)$ term, we can easily obtain the solution to (29) for $a_{\mu}^{a}$ in terms of $n^{a}$,

$$
a_{\mu}^{a}=C_{\mu} n^{a}-\varepsilon^{a b c} n^{b} \partial_{\mu} n^{c},
$$

where we have introduced a vector field $C_{\mu}$ which is arbitrary at this stage. This solution demonstrates the clear separation of the gauge fields:

$$
a_{\mu}^{\|}=C_{\mu} n^{a} \quad \text { and } \quad a_{\mu}^{\perp}=-\varepsilon^{a b c} n^{b} \partial_{\mu} n^{c} .
$$

The relation for the broken components, $a_{\mu}^{\perp}$, is nothing but a non-Abelian analogue of the relation between the gauge field and the quantum phase of Cooper pair, $A_{\mu}=\partial_{\mu} \theta$, in superconductivity, and also corresponds to the MaurerCartan one-form of $G / H \sim S U(2) / U(1)$ in terms of the
Nambu-Goldstone modes $n^{a}$ [20,21]. Requiring the matching condition to the static solution (17), $a_{0}^{a}=\mu_{s} \delta^{a 3}$ and $a_{i}^{a}=0$ for $n^{a}=(0,0,1)$, the vector field $C_{\mu}$ should satisfy the condition:

$$
C_{0}=\mu_{s} \quad \text { and } \quad C_{i}=0,
$$

in the static and homogeneous limit. Note that the relation (31) and the ansatz (18) do not induce new contributions of the scalar fields to the energy-momentum tensor $T_{M N}$ in the Einstein equations, and consequently the analysis in the probe approximation remain intact.

Next, we consider the effective Yang-Mills equations, (26) and (27). It is not difficult to show that the relation (31) leads to vanishing currents $J_{\mu}^{a}$ up to $\mathcal{O}\left(u^{2}\right)$, using the explicit form (25). Furthermore, the ansatz for gauge fields (19) with $A_{u}^{a}=0$ implies

$\partial_{u}\left(u^{-1} F_{u 0}^{a}\right)=0 \quad$ and $\quad \partial_{u}\left(u^{-1} f F_{u i}^{a}\right)=0+\mathcal{O}\left(u^{4}\right)$.

Dropping the higher order terms such as $\mathcal{O}\left(u^{4}\right)$, the remaining Yang-Mills equations reduce to

$$
D_{i} F_{i 0}^{a}=0 \quad \text { and } \quad D_{0} F_{0 i}^{a}+D_{j} F_{j i}^{a}=0 .
$$

From the viewpoint of the boundary theory (on the ferromagnet side), the first equation corresponds to a non-Abelian version of Gauss's law, and the second corresponds to a non-Abelian version of Ampere's law without source and currents, for the spin gauge fields [22]. Using the relation (31), we obtain the $S U(2)$ field strength, ${ }^{4}$

$F_{\mu \nu}^{a}=n^{a}\left[\left(\partial_{\mu} C_{\nu}-\partial_{\nu} C_{\mu}\right)-\varepsilon^{b c d} n^{b} \partial_{\mu} n^{c} \partial_{\nu} n^{d}\right] \equiv n^{a} f_{\mu \nu}$.

Note that a component of the field strength, $f_{\mu \nu}$, parallel to the magnetization $n^{a}$ only remains. With the field strength (36), the effective Yang-Mills equations (35) and the Bianchi identity for the $S U(2)$ gauge field are reduced to the following equations:

$$
\partial_{\mu} f_{\mu \nu}=0 \quad \text { and } \quad \epsilon^{\mu \nu \rho \sigma} \partial_{\nu} f_{\rho \sigma}=0 .
$$

The above equations are the same form as the Maxwell equations, and the terms depending on $n^{a}$ in the gauge field $f_{\mu \nu}$ actually corresponds to the so-called spin electromagnetic field discussed in the study on ferromagnetic metals $[22,23]$. The gauge field (36) also corresponds to the unbroken $U(1)$ gauge field upon the symmetry breaking from $S U(2)$ to $U(1)$, with a space-dependent order parameter, which is frequently discussed in the context of

\footnotetext{
${ }^{4}$ We used the relation $\varepsilon^{a b c} \partial_{\mu} n^{b} \partial_{\nu} n^{c}=n^{a} \varepsilon^{b c d} n^{b} \partial_{\mu} n^{c} \partial_{\nu} n^{d}$ due to $\sum_{a} n^{a} n^{a}=1$.
} 
solitonic monopoles in non-Abelian gauge theories [24]. Since the unbroken gauge fields in the holographic dual theory are identified as the (exactly) conserved currents in the boundary quantum system, the gauge field $C_{\mu}$ is naturally identified as the spin current with the polarization parallel to the magnetization $n^{a}$, which originates from conduction electrons.

So far, we have obtained the relation between the gauge field $a_{\mu}^{a}$ and the (normalized) magnetization $n^{a}$, which implies that the gauge field dynamics can be solely reduced to the dynamics of the magnetization and the spin electromagnetic field $C_{\mu}$. Finally, we consider the remaining Yang-Mills equation in the radial $u$-direction, in the holographic dual theory:

$$
\frac{1}{\sqrt{-g}} \partial_{\mu}\left(\sqrt{-g} F^{\mu и a}\right)+\epsilon^{a b c} A_{\mu}^{b} F^{\mu u c}=J^{u a} .
$$

This equation is derived by the variation of the radial $u$-component of the $S U(2)$ gauge fields and specifies the dynamics of the gauge fields in the five-dimensional bulk; this equation cannot be seen in the ferromagnetic system on the boundary. With the ansatz (18), the radial component of the current also vanishes $\left(J_{u}^{a} \equiv 0\right)$, and the gauge fixing condition $A_{u}^{a} \equiv 0$ leads to the simple $S U(2)$ field strength $F_{\mu u}^{a}=-\partial_{u} A_{\mu}^{a}$ such as

$$
\begin{aligned}
F_{0 u}^{\|} & =2 u a_{0}^{\|}(t, x), \quad F_{0 u}^{\perp}=2 u a_{0}^{\perp}(t, x), \\
F_{i u}^{\|} & =2 u \sigma_{s}^{\|} a_{i}^{\|}(t, x), \quad F_{i u}^{\perp}=-2 u \sigma_{s}^{\perp} a_{i}^{\perp}(t, x),
\end{aligned}
$$

where we used the ansatz (19) and discarded the irrelevant $\mathcal{O}\left(u^{3}\right)$ terms. From these forms, the second term in the lefthand side of (38) automatically vanishes due to $\varepsilon^{a b c} a_{\mu}^{b} a_{\mu}^{c}=0$. Inserting the forms of field strength (39) and the relation (31), the equation can be recast as the following form:

$\partial_{0}\left(C_{0} n^{a}-\varepsilon^{a b c} n^{b} \partial_{0} n^{c}\right)-\partial_{i}\left(\sigma_{s}^{\|} C_{i} n^{a}+\sigma_{s}^{\perp} \varepsilon^{a b c} n^{b} \partial_{i} n^{c}\right)=0$,

where the subleading terms are neglected. Here, we can write down the effective equation of motion for the magnetization $n^{a}$ in our holographic dual model:

$$
C_{0} \dot{n}^{a}-\varepsilon^{a b c} n^{b} \ddot{n}^{c}=\sigma_{s}^{\perp} \varepsilon^{a b c} n^{b} \nabla^{2} n^{c}+\sigma_{s}^{\|} C_{i} \partial_{i} n^{a},
$$

where the dot denotes the time derivative and $\nabla^{2}=\partial_{i} \partial_{i}{ }^{5}$ Here, we consider the condition, $\partial_{0} C_{0}-\sigma_{s}^{\|} \partial_{i} C_{i}=0$, on the unbroken gauge field due to the constraint

\footnotetext{
${ }^{5} \mathrm{~A}$ similar analysis on effective equations at the linearized level in two-dimensional magnetic systems has been also discussed in Ref. [12].
}

$\sum_{a} n^{a} n^{a}=1$. This condition implies the conservation of the spin current of conduction electrons, which corresponds to the unbroken gauge field $C_{\mu}$, as seen below. Note that, since the Maxwell equations (37) for $C_{\mu}$ is gauge invariant, this condition can be consistently imposed as a gauge fixing condition.

Considering the matching condition (33), we decompose $C_{0}$ into $C_{0}=\mu_{s}+\tilde{C}_{0}$. Finally, we obtain the holographic equation for magnetization dynamics:

$\mu_{s} \dot{n}^{a}-\varepsilon^{a b c} n^{b} \ddot{n}^{c}=\sigma_{s}^{\perp} \varepsilon^{a b c} n^{b} \nabla^{2} n^{c}-\tilde{C}_{0} \dot{n}^{a}+\sigma_{s}^{\|} C_{i} \partial_{i} n^{a}$.

Here, we take the spin chemical potential to be $\mu_{s}=$ $-M_{s} / \gamma$ with the magnitude of spontaneous magnetization $M_{s}$ and the gyromagnetic ratio $\gamma(>0),{ }^{6}$ and also identify the spin current and spin accumulation due to conduction electrons as $J_{s i}^{\|}=-\sigma_{s}^{\|} C_{i}$ and $\Delta \mu_{s}=\tilde{C}_{0}$, using the holographic dictionary. Then, the holographic equation becomes the same form as the Landau-Lifshitz equation (without damping terms),

$\frac{M_{s}}{\gamma} \dot{n}^{a}+\varepsilon^{a b c} n^{b} \ddot{n}^{c}=-\sigma_{s}^{\perp} \varepsilon^{a b c} n^{b} \nabla^{2} n^{c}+\Delta \mu_{s} \dot{n}^{a}+J_{s i}^{\|} \partial_{i} n^{a}$.

The last two terms in the right-hand side can be interpreted as the well-known terms from spin transfer torque, which describes the transfer of spin angular momentum between localized magnetic moments and conduction electrons $[22]^{7}$ Furthermore, the holographic Landau-Lifshitz equation (42) also naturally incorporate the so-called spin inertia term proportional to the second time derivative of the magnetization. ${ }^{8}$ This spin inertia term originates from interactions between localized magnetic moments and environments such as conduction electrons, and it has been discussed in metallic ferromagnets as a subleading correction term in the low energy expansion. (See Ref. [26] and references therein.) Note that the holographic equation corresponds to the effective equation of motion up to the second order in the derivative expansion, where the quantities are expanded in powers of the time and spatial derivative, and it describes the low energy magnetization dynamics $\left(E / \mu_{s}<1\right)$ with the long wave length.

It should be also noted that the holographic LandauLifshitz equation automatically incorporates the spin

\footnotetext{
${ }^{6}$ The negative sign is introduced due to the negative value of the gyromagnetic ratio for electrons.

${ }^{7}$ Another higher dimensional perspective on spin transfer torque has been recently discussed in Ref. [25].

${ }^{8}$ The coefficient of the spin inertia term generally depends on the gauge coupling $g$, and can be modified due to the nonlinear effect of the fluctuation dynamics.
} 
transfer torque due to conduction electrons without introducing the corresponding fields to electrons in the dual gravitational theory. This is consistent with the thermodynamical results at low temperatures, which was obtained in Ref. [11].

\section{PHENOMENOLOGY OF HOLOGRAPHIC MAGNETIZATION DYNAMICS}

In the isotropic ferromagnets sufficiently below the Curie temperature $\left(T \ll T_{c}\right)$, the dynamics of magnetization vector (or density of magnetic moments), $M^{a}$, is described by the Landau-Lifshitz equation $[1,27]$ :

$$
\frac{\partial M^{a}}{\partial t}=-\alpha \epsilon^{a b c} M^{b} \nabla^{2} M^{c}
$$

with $\sum_{a=1}^{3} M^{a} M^{a}=M(T)^{2}=$ const. In the following discussion, the external magnetic field and the damping term (or relaxation term) are ignored for simplicity. From the quadratic constraint, the magnetization vector can be represented as $M^{a}(x, t)=M(T) n^{a}(x, t)$ with the unit vector $n^{a}(x, t)$. In terms of $n^{a}(x, t)$, the Landau-Lifshitz equation becomes

$$
M(T) \frac{\partial n^{a}}{\partial t}=-\alpha M(T)^{2} \epsilon^{a b c} n^{b} \nabla^{2} n^{c} .
$$

Note that the equation has two parameters, the magnitude of spontaneous magnetization, $M(T)$, at the temperature $T$, and the spin stiffness constant, $\alpha$.

Comparing the holographic equation (42) with the Landau-Lifshitz equation (45), we find that the spin chemical potential, $\mu_{s}$, in the gauge field solution (17) should be proportional to the magnitude of magnetization, and the spin stiffness constant is given by the coefficients $\sigma_{s}^{\perp}$ in the gauge field solution (20) in the following way:

$$
\mu_{s} \propto-M(T) \text { and } \sigma_{s}^{\perp} \propto \alpha M(T)^{2} .
$$

In our holographic dual model, the magnitude of magnetization, $M(T)$, at the temperature $T$ is given by the static solution of the scalar field $\Phi(u)$ through the formula (14). The first relation between the magnitude of magnetization and the spin chemical potential in ferromagnets is well known, and frequently used as the starting point to analyze the various spintronic phenomena [2].

Although the spin chemical potential in the equilibrium, $\mu_{s}$, is an integration constant, the coefficient, $\sigma_{s}^{\perp}$, is the derived quantity from the gauge field equation, and thus the second relation in (46) on the spin stiffness constant is a nontrivial consequence in the holographic dual model. In order to obtain the coefficient, $\sigma_{s}^{\perp}$, we consider the linearized equation of motion for gauge fields on the background solution, with the static and homogeneous ansatz, $A_{i}^{\perp}=k G^{\perp}(u)$, where $k=$ const. $^{9}$ Inserting this ansatz into the Yang-Mills equation (27), we have the following linearized equation for $G^{\perp}(u)$ :

$$
u^{3} \frac{d}{d u}\left(\frac{f(u)}{u}\left(\frac{d G^{\perp}}{d u}\right)\right)+\frac{\left(u a^{3}(u)\right)^{2}}{f(u)} G^{\perp}=0,
$$

where the metric (8) and the $S U(2)$ gauge field (17) are assumed to be the background. Note that this is a linear equation for $G^{\perp}$, and the constant $k$ is irrelevant. Here, we impose the first relation in (46), $\mu_{s}=-M(T) / M(0)$, which is the magnetization normalized by the saturated magnetization, $M(T=0) .{ }^{10}$ Using the numerical results of the holographic spontaneous magnetization, $M(T)$ in [11], which is obtained using the scalar potential $V(|\phi|)=$ $\lambda\left(|\phi|^{2}-m^{2} / \lambda\right)^{2} / 4$ with $\lambda=1$ and $m^{2}=35 / 9$, we can numerically solve the Eq. (47) and obtain the asymptotic expansion $(20)$ near the boundary $(u \sim 0)$. The numerical results of temperature dependences of the spin-wave stiffness, $D(T) \simeq \sigma_{s}^{\perp} / M(T)$, which appears in the dispersion relation of spin waves, $\omega=D(T) k^{2}$, and the spin stiffness constant, $\alpha(T) \simeq \sigma_{s}^{\perp} / M(T)^{2}$, are shown in Fig. 1 .

The results on the spin-wave stiffness in Fig. 1(a) clearly show that $D(T) \propto M(T)$, which is consistent with the relation (46) based on the Landau-Lifshitz equation (44). Furthermore, the results in Fig. 1(b) imply the slight temperature dependence of the spin stiffness constant, $\alpha=\alpha(T)$, which can be attributed to the nonlinear spinwave effects [28].

A similar argument also holds for the unbroken (or parallel) component of the gauge fields, $A_{i}^{\|}$, and we can obtain the coefficient $\sigma_{s}^{\|}$, which leads to the spin torque term in the holographic Landau-Lifshitz equation (42). The nontrivial profile of gauge field, $A_{x}^{\|}(u)$, which is the parallel component to the spin chemical potential, $A_{0}^{\|}$, leads to the nonvanishing off-diagonal contribution in the right-hand side of the Einstein equation (2), and thus induces the fluctuation of the metric $g_{t x}(u)=h_{t x}(u) / u^{2}$, where $h_{t x}(u)$ parametrizes the fluctuation finite on the boundary. At the linearized level, two fluctuations, $A_{x}^{\|}(u)$ and $h_{t x}(u)$, form the closed equations, which come from the Yang-Mills equation and Einstein equation, respectively [29,30]:

$$
\begin{aligned}
u \frac{d}{d u}\left(\frac{f(u)}{u}\left(\frac{d A_{x}^{\|}}{d u}\right)\right)+\left(\frac{d a^{3}(u)}{d u}\right) \frac{d}{d u}\left(u^{2} h_{t x}\right) & =0 \\
u^{-2} \frac{d}{d u}\left(u^{2} h_{t x}\right)+2\left(\frac{d a^{3}(u)}{d u}\right) A_{x}^{\|} & =0 .
\end{aligned}
$$

\footnotetext{
${ }^{9}$ The nontrivial profile $A_{i}^{\perp}(u)$ on the background does not contribute to the energy-momentum tensor in the Einstein equation at the linearized level.

${ }^{10}$ The proportionality constant is chosen for convenience in numerical calculations.
} 


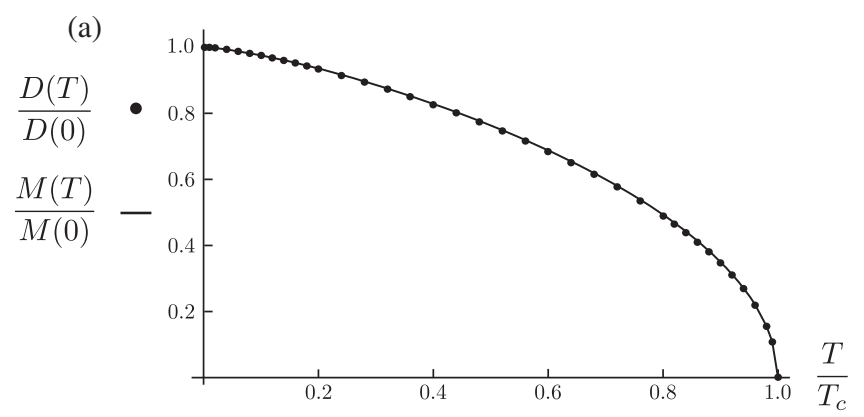

(b)

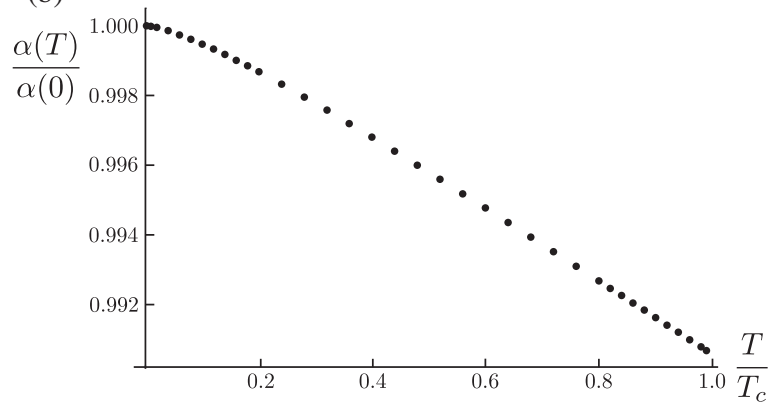

FIG. 1. Temperature dependence of the spin-wave stiffness is shown in Fig. (a): The dots are numerical results for $D(T) / D(0)$, and the bold line is the magnetization curve, $M(T) / M(0)$. Temperature dependence of the spin stiffness constant, $\alpha(T) / \alpha(0)$, is shown in Fig. (b). All the results are calculated with the parameters, $\lambda=1$ and $m^{2}=35 / 9$.

Deleting the metric fluctuation, we can obtain the equation for $G^{\|}(u)$ :

$$
u \frac{d}{d u}\left(\frac{f(u)}{u}\left(\frac{d G^{\|}}{d u}\right)\right)-2 u^{2}\left(\frac{d a^{3}(u)}{d u}\right)^{2} G^{\|}=0 .
$$

We can numerically solve the equation, and obtain the coefficient $\sigma_{s}^{\|}$from the asymptotic expansion of the solution in (20). The resulting temperature dependence of the spin torque coefficient, $\tau_{s}(T)=\sigma_{s}^{\|} / M(T)$, is shown in Fig. 2 .

The results on the magnitude of the spin transfer torque, $\tau_{s}(T)$, show that the spin torque effect is approximately

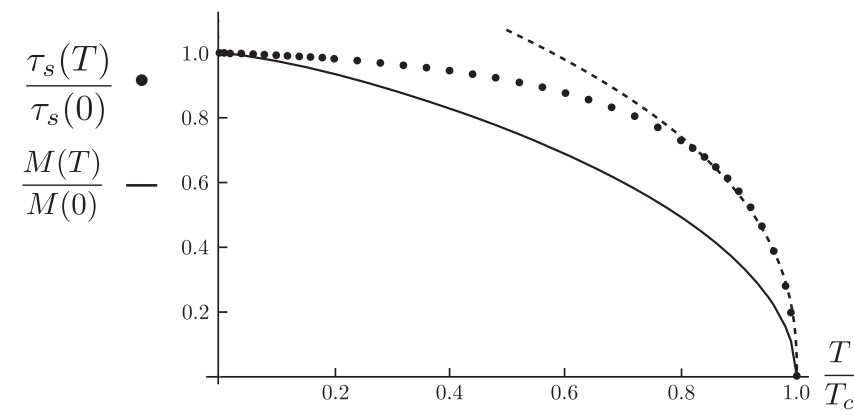

FIG. 2. Temperature dependence of the spin torque coefficient: The dots are numerical results for $\tau_{s}(T) / \tau_{s}(0)$, and the bold line is the magnetization curve $M(T) / M(0)$. The dashed line is the fitting curve, $\tau_{s}(T) / \tau_{s}(0)=c\left(1-T / T_{c}\right)^{2 / 5}$ with $c \simeq 1.41$, near the Curie temperature. constant at low temperatures (in comparison with magnetization curve), and is vanishing towards the Curie temperature as $\tau_{s}(T) \propto\left(1-T / T_{c}\right)^{2 / 5}$. This property at low temperatures is consistent with the phenomenological form of the spin transfer torque, $\left(\boldsymbol{J}_{s}^{\|} \cdot \nabla n^{a}\right) / M(T)$, whose magnitude is independent of the norm of magnetization due to $\left|\boldsymbol{J}_{s}^{\|}\right| \propto M(T)$ at the leading order [22]. In addition, the finite spin torque coefficient is a consequence of the both fluctuations of the gauge field and metric. In accordance with the holographic dictionary [29], the metric fluctuation $h_{t x}$ corresponds to the temperature gradient, $\nabla_{x} T / T$, in the ferromagnetic system. This calculation implies that the effect of spin transfer torque appears only in the nonequilibrium situations, where spin transfer is accompanied by heat (or entropy) transfer.

\section{SUMMARY AND DISCUSSION}

We have discussed a novel approach to understand magnetization dynamics in ferromagnets using the holographic realization of ferromagnetic systems. The LandauLifshitz equation describing magnetization dynamics was derived from the Yang-Mills-Higgs equations in the dual gravitational theory. This holographic Landau-Lifshitz equation automatically incorporates not only the exchange interaction but also the spin transfer torque effect due to conduction electrons. Furthermore, we numerically investigated the temperature dependences of the spin-wave stiffness and the magnitude of spin transfer torque in the holographic dual theory, and the results obtained so far are consistent with the known properties of magnetization dynamics in ferromagnets with conduction electrons.

This holographic approach to magnetization dynamics can be applied to more generic situations. For instance, the holographic Landau-Lifshitz equation can incorporate the damping term by considering more generic metric fluctuations, which correspond to phonon dynamics in the boundary ferromagnets. Moreover, the holographic dual theory may provide geometric approaches to spin caloritronics [31], where magnetization dynamics is considered under temperature gradients, from higher dimensional perspectives. We thus believe that the holographic approach provides useful tools to analyze nonequilibrium and nonlinear dynamics of magnetization in ferromagnets, and also leads to new perspectives in spintronics from gravitational physics.

\section{ACKNOWLEDGMENTS}

The authors thank M. Ishihara for collaboration at the early stage of this work, and also K. Harii and Y. Oikawa for useful discussions. The works of N. Y. and E. S. were supported in part by Grant-in Aid for Scientific Research on Innovative Areas "Nano Spin Conversion Science" (26103005), and the work of K. S. was supported in part by JSPS KAKENHI Grant No. JP17H06460. The works of N. Y. and E. S. were supported in part by ERATO, JST. 
[1] L. D. Landau and E. M. Lifshitz, On the theory of the dispersion of magnetic permeability in ferromagnetic bodies, Phys. Z. Sowjetunion 8, 153 (1935); Reprinted in Perspectives in Theoretical Physics, edited by L.P. Pitaevski (Pergamon Press, Oxford, England, 1992).

[2] S. Maekawa, S. O. Valenzuela, E. Saitoh, and T. Kimura, Spin Current, 2nd ed. (Oxford University Press, New York, 2017), Vol. 22.

[3] L. Berger, Emission of spin waves by a magnetic multilayer traversed by a current, Phys. Rev. B 54, 9353 (1996).

[4] J.C. Slonczewski, Current-driven excitation of magnetic multilayers, J. Magn. Magn. Mater. 159, L1 (1996).

[5] Y. Tserkovnyak, A. Brataas, and G. E. W. Bauer, Enhanced Gilbert Damping in Thin Ferromagnetic Films, Phys. Rev. Lett. 88, 117601 (2002).

[6] J. M. Maldacena, The large $N$ limit of superconformal field theories and supergravity, Int. J. Theor. Phys. 38, 1113 (1999); Adv. Theor. Math. Phys. 2, 231 (1998).

[7] S. S. Gubser, I. R. Klebanov, and A. M. Polyakov, Gauge theory correlators from noncritical string theory, Phys. Lett. B 428, 105 (1998).

[8] E. Witten, Anti-de Sitter space and holography, Adv. Theor. Math. Phys. 2, 253 (1998).

[9] J. Zaanen, Y. Liu, Y. W. Sun, and K. Schalm, Holographic Duality in Condensed Matter Physics (Cambridge University Press, Cambridge, England, 2015).

[10] S. A. Hartnoll, A. Lucas, and S. Sachdev, Holographic Quantum Matter (MIT Press, Cambridge, 2018).

[11] N. Yokoi, M. Ishihara, K. Sato, and E. Saitoh, Holographic realization of ferromagnets, Phys. Rev. D 93, 026002 (2016).

[12] N. Iqbal, H. Liu, M. Mezei, and Q. Si, Quantum phase transitions in holographic models of magnetism and superconductors, Phys. Rev. D 82, 045002 (2010).

[13] R. G. Cai and R. Q. Yang, Paramagnetism-ferromagnetism phase transition in a dyonic black hole, Phys. Rev. D 90, 081901(R) (2014).

[14] R. G. Cai, R. Q. Yang, Y. B. Wu, and C. Y. Zhang, Massive 2-form field and holographic ferromagnetic phase transition, J. High Energy Phys. 11 (2015) 021.

[15] V. E. Hubeny and M. Rangamani, A holographic view on physics out of equilibrium, Adv. High Energy Phys. 2010, 297916 (2010).
[16] C. N. Yang and R. L. Mills, Conservation of isotopic spin and isotopic gauge invariance, Phys. Rev. 96, 191 (1954).

[17] C. P. Herzog and S. S. Pufu, The second sound of SU(2), J. High Energy Phys. 04 (2009) 126.

[18] V. Balasubramanian, P. Kraus, A. E. Lawrence, and S. P. Trivedi, Holographic probes of anti-de Sitter space-times, Phys. Rev. D 59, 104021 (1999).

[19] I. R. Klebanov and E. Witten, AdS/CFT correspondence and symmetry breaking, Nucl. Phys. B556, 89 (1999).

[20] H. Leutwyler, Nonrelativistic effective Lagrangians, Phys. Rev. D 49, 3033 (1994).

[21] J. M. Román and J. Soto, Effective field theory approach to ferromagnets and antiferromagnets in crystalline solids, Int. J. Mod. Phys. B 13, 755 (1999).

[22] G. Tatara, Effective gauge field theory of spintronics, Physica (Amsterdam) 106E, 208 (2019).

[23] G. E. Volovik, Linear momentum in ferromagnets, J. Phys. C 20, L83 (1987).

[24] For a review, J. A. Harvey, Magnetic monopoles, duality and supersymmetry, in Trieste 1995, High Energy Physics and Cosmology (World Scientific Publishing, Singapore, 1996).

[25] S. Dasgupta and O. Tchernyshyov, Energy-momentum tensor of a ferromagnet, Phys. Rev. B 98, 224401 (2018).

[26] T. Kikuchi and G. Tatara, Spin dynamics with inertia in metallic ferromagnets, Phys. Rev. B 92, 184410 (2015).

[27] E. M. Lifshitz and L. P. Pitaevskii, Statistical Physics: Theory of the Condensed State (Elsevier, New York, 2013), Vol. 9.

[28] U. Atxitia, D. Hinzke, O. Chubykalo-Fesenko, U. Nowak, H. Kachkachi, O. N. Mryasov, R. F. Evans, and R.W. Chantrell, Multiscale modeling of magnetic materials: Temperature dependence of the exchange stiffness, Phys. Rev. B 82, 134440 (2010).

[29] S. A. Hartnoll, Lectures on holographic methods for condensed matter physics, Classical Quantum Gravity 26, 224002 (2009).

[30] C. P. Herzog, K. W. Huang, and R. Vaz, Linear resistivity from non-Abelian black holes, J. High Energy Phys. 11 (2014) 066.

[31] G. E. W. Bauer, E. Saitoh, and B. J. Van Wees, Spin caloritronics, Nat. Mater. 11, 391 (2012). 